\title{
REAL-LINEAR OPERATORS ON QUATERNIONIC HILBERT SPACE
}

\author{
N. C. POWERS ${ }^{1}$
}

\begin{abstract}
The main result is that any continuous real-linear operator $A$ on a quaternionic Hilbert space has a unique decomposition $A=A_{0}+i_{1} A_{1}+i_{2} A_{2}+i_{3} A_{3}$, where the $A_{v}$ are continuous linear operators and $\left(i_{1}, i_{2}, i_{3}\right)$ is any right-handed orthonormal triad of vector quaternions. Other results concern the place of the colinear and complex-linear operators in this characterisation and the effect on the $A_{\nu}$ of a rotation of the triad of vector quaternions. A new result concerning symplectic images of a quaternionic Hilbert space is also presented.
\end{abstract}

1. Introduction. The study of group representations on quaternionic Hilbert space, reviewed in [1], makes extensive use of the complex Hilbert space structure that can be imposed on a quaternionic Hilbert space. This is also true of the recent work by Viswanath [3] on normal linear operators. In the present work the real Hilbert space structure is also considered, with particular attention to real-linear operators. The main result is that the class of continuous real-linear operators on a quaternionic Hilbert space $\mathscr{H}$ is simply the quaternion algebra generated by the real algebra of continuous linear operators on $\mathscr{H}$.

The author wishes to thank his Ph.D. supervisor, Professor B. Abrahamson of the Flinders University of South Australia, for his advice and encouragement while this research was being carried out. The hospitality of the University of Cambridge, where this paper was largely written, is gratefully acknowledged.

2. Preliminaries. The division ring $\mathscr{Q}$ of quaternions is the real algebra generated by the elements of the quaternion group $\{ \pm 1, \pm i, \pm j, \pm k\}$. Thus any quaternion $q$ can be written in the form:

$$
q=q_{0}+q_{1} i+q_{2} j+q_{3} k \quad\left(q_{v} \in R ; v=0,1,2,3\right) .
$$

Received by the editors November 29, 1972.

AMS (MOS) subject classifications (1970). Primary 15A33, 47-00, 47H99.

Key words and phrases. Quaternions, quaternionic Hilbert space, quaternionic matrix, real-linear operator, symplectic image.

${ }^{1}$ The research for this paper was carried out at the Flinders University of South Australia with the support of a Commonwealth Postgraduate award and subsequently of a Flinders University Research Scholarship. 
The elements of the real subspace of $\mathscr{Q}$ generated by 1 are called real quaternions, while the elements of the subspace generated by $\{i, j, k\}$ are called vector quaternions. Since the latter subspace can be identified with $R^{3}$ we often write $q=q_{0}+\xi\left(\xi \in R^{3}\right)$ or $q=\sum_{v=0}^{3} q_{v} i_{v}$, where $i_{0}=1$ and $\left(i_{1}, i_{2}, i_{3}\right)$ is any right-handed orthonormal triad in $\boldsymbol{R}^{3}$. Such a triad is called a quaternion basis.

The relationship between two quaternion bases $\left(i_{1}, i_{2}, i_{3}\right)$ and $\left(i_{1}^{\prime}, i_{2}^{\prime}, i_{3}^{\prime}\right)$ can be expressed by means of a $3 \times 3$ real orthogonal matrix $L=\left[l_{\mu v}\right]$, viz: $\left[i_{1}^{\prime} i_{2}^{\prime} i_{3}^{\prime}\right]=\left[i_{1} i_{2} i_{3}\right] L$, i.e. $i_{\mu}^{\prime}=\sum_{\lambda=1}^{3} i_{\lambda} l_{\lambda \mu}(\mu=1,2,3)$.

Thus, if $q=\sum_{v=0}^{3} q_{v} i_{v}=\sum_{v=0}^{3} q_{v}^{\prime} i_{v}^{\prime}$, then $\left[q_{0}^{\prime}, q_{1}^{\prime}, q_{2}^{\prime}, q_{3}^{\prime}\right]=\left[q_{0}, q_{1}, q_{2}, q_{3}\right] M$, where $M$ is the $4 \times 4$ matrix

$$
\left[\begin{array}{c:c}
1 & 0 \\
\hdashline 0 & L
\end{array}\right] \text {. }
$$

Alternatively, the rotation in $\boldsymbol{R}^{3}$ defined by $L$ can be represented by an inner automorphism $\omega$ of $\mathscr{Q}$, viz: $i_{\mu}^{\prime}=q i_{\mu} q^{-1}(\mu=1,2,3)$, where $q$ is a unit quaternion. This follows from the well-known fact that the continuous automorphisms of the division ring $\mathscr{Q}$ are all inner automorphisms and are in one-to-one correspondence with the rotations in $\boldsymbol{R}^{3}$.

The canonical anti-automorphism $\sigma$ of $\mathscr{Q}$, called quaternion conjugation, is defined by

$$
q^{\sigma}=\left(q_{0}+\xi\right)^{\sigma}=q_{0}-\xi, \quad \forall q \in \mathscr{Q} .
$$

3. Real and complex Hilbert space structures. Let $\mathscr{H}$ be a quaternionic Hilbert space with inner product $\langle$,$\rangle . It will sometimes be convenient to$ decompose this inner product as follows:

$$
\langle x, y\rangle=\sum_{v=0}^{3} i_{v}\langle x, y\rangle_{v}, \quad \forall x, y \in \mathscr{H},
$$

where $\langle x, y\rangle_{v} \in \boldsymbol{R}(\nu=0,1,2,3)$. It is easy to verify that $\langle x, y\rangle_{v}=\left\langle x, i_{v} y\right\rangle_{0}$ $(\nu=1,2,3)$.

It is well known (see, for example [3]) that $\mathscr{H}$ can be given the structure of a complex Hilbert space. This is done by identifying the complex field $\boldsymbol{C}$ with the subfield $\boldsymbol{C}\left(i_{1}\right)$ of $\mathscr{Q}$, where $\boldsymbol{C}\left(i_{1}\right)=\left\{q_{0}+q_{1} i_{1}: q_{0}, q_{1} \in \boldsymbol{R}\right\}$, and defining the complex inner product $($,$) by (x, y)=\langle x, y\rangle_{0}+i_{1}\langle x, y\rangle_{1}$, $\forall x, y \in \mathscr{H}$. The resulting complex Hilbert space $\mathscr{H}_{1}\left(i_{1}\right)$ is called the symplectic image of $\mathscr{H}$. It can be verified that, if $S$ is an orthonormal basis for $\mathscr{H}$, then the set $S_{1}=\left\{y, i_{2} y: y \in S\right\}$ is an orthonormal basis for $\mathscr{H}_{1}\left(i_{1}\right)$.

The notation $\mathscr{H}_{1}\left(i_{1}\right)$ has been chosen to emphasise the fact that the complex inner product depends on the choice of unit vector quaternion $i_{1}$. However, there is a simple relationship between any two complex inner products defined in this way. 
THEOREM 1. Let $u$ and $u^{\prime}$ be two unit vector quaternions and let (, ) and $(,)^{\prime}$ be the natural images in $C$ of the inner products on $\mathscr{H}_{1}(u)$ and $\mathscr{H}_{1}\left(u^{\prime}\right)$ respectively. If $q$ is a unit quaternion such that $u^{\prime}=q u q^{\sigma}$, then

$$
(x, y)^{\prime}=\left(q^{\sigma} x, q^{\sigma} y\right), \quad \forall x, y \in \mathscr{H} .
$$

Proof. For each $x, y \in \mathscr{H}$ we have

$$
(x, y)^{\prime}=\langle x, y\rangle_{0}+i\left\langle x, u^{\prime} y\right\rangle_{0} .
$$

But

$$
\begin{aligned}
\left\langle x, u^{\prime} y\right\rangle_{0} & =\left\langle x, q u q^{\sigma} y\right\rangle_{0}=\operatorname{Re}\left[\left\langle x, u q^{\sigma} y\right\rangle q^{\sigma}\right] \\
& =\operatorname{Re}\left[q^{\sigma}\left\langle x, u q^{\sigma} y\right\rangle\right]=\left\langle q^{\sigma} x, q^{\sigma} y\right\rangle_{0} .
\end{aligned}
$$

Also, using the fact that

$$
\operatorname{Re}(a b c)=\operatorname{Re}(b c a)=\operatorname{Re}(c a b), \quad \forall a, b, c \in \mathscr{Q},
$$

we have $\langle x, y\rangle_{0}=\operatorname{Re}\left[\langle x, y\rangle q q^{\sigma}\right]=\operatorname{Re}\left[q^{\sigma}\langle x, y\rangle q\right]=\left\langle q^{\sigma} x, q^{\sigma} y\right\rangle_{0}$. Substitution in (2) gives (1).

This result has not appeared in the literature to date.

It is also possible to give $\mathscr{H}$ the structure of a real Hilbert space $\mathscr{H}_{\mathbf{0}}$. The real-bilinear form $\langle,\rangle_{0}$ on $\mathscr{H}$ defined by $\langle x, y\rangle_{0}=\operatorname{Re}\langle x, y\rangle, \forall x, y \in$ $\mathscr{H}$, satisfies the axioms for an inner product on a real Hilbert space.

If $S$ is an orthonormal basis for $\mathscr{H}$, then the set $S_{0}=\left\{i_{\mu} y: y \in S, \mu=\right.$ $0,1,2,3\}$ is an orthonormal basis for $\mathscr{H}_{0}$.

4. Classes of operators. The fact that it is possible to impose real and complex Hilbert space structures on the underlying set of a quaternionic Hilbert space makes it possible to assign more than one meaning to the adjective "linear".

Definition 1. Let $\mathscr{H}$ and $\mathscr{K}$ be quaternionic Hilbert spaces and let $A: \mathscr{H} \rightarrow \mathscr{K}$ be an operator such that

$$
A(x+y)=A x+A y, \quad \forall x, y \in \mathscr{H} .
$$

$\boldsymbol{A}$ is called real-linear or $\boldsymbol{R}$-linear, if for each real quaternion $q$,

$$
A(q x)=q A x, \quad \forall x \in \mathscr{H} .
$$

$A$ is called $C\left(i_{1}\right)$-linear if (4) holds for each $q$ in $C\left(i_{1}\right)$, linear if (4) holds for every quaternion $q$.

The classes of continuous real-linear, continuous $C\left(i_{1}\right)$-linear, and continuous linear operators from $\mathscr{H}$ to $\mathscr{K}$ are denoted by $\mathscr{L}\left(\mathscr{H}_{0}, \mathscr{K}_{0}\right)$, $\mathscr{L}\left(\mathscr{H}_{1}\left(i_{1}\right), \mathscr{K}_{1}\left(i_{1}\right)\right)$, and $\mathscr{L}(\mathscr{H}, \mathscr{K})$ respectively; in the case where $\mathscr{H}=$ $\mathscr{K}$ the abbreviations $\mathscr{L}\left(\mathscr{H}_{0}\right), \mathscr{L}\left(\mathscr{H}_{1}\left(i_{1}\right)\right)$, and $\mathscr{L}(\mathscr{H})$ are used. 
Definition 2. An operator $A: \mathscr{H} \rightarrow \mathscr{K}$ is called colinear if (3) holds and there is a fixed automorphism $\omega$ of $\mathscr{Q}$ such that, for each quaternion $q$, $A(q x)=q^{\omega} A x, \forall x \in \mathscr{H}$.

When we wish to emphasise the particular automorphism $\omega$ associated with $A$ we say that $A$ is $\omega$-linear. The class of continuous $\omega$-linear operators from $\mathscr{H}$ to $\mathscr{K}$ is denoted by $\mathscr{L}_{\omega}(\mathscr{H}, \mathscr{K})$.

It is well known (see, for example, [1]) that every colinear operator is a quaternion multiple of a linear operator. More precisely, given any nonzero quaternion $a$, let $\omega(a)$ denote the automorphism $\omega(a): q \mapsto a q a^{-1}$ of $\mathscr{Q}$. Then

$$
\mathscr{L}_{\omega(a)}(\mathscr{H}, \mathscr{K})=\{a T: T \in \mathscr{L}(\mathscr{H}, \mathscr{K})\} \text {. }
$$

Definition 3. The adjoint of a continuous real-linear operator $A$ : $\mathscr{H} \rightarrow \mathscr{H}$ is the unique continuous real-linear operator $A^{*}: \mathscr{H} \rightarrow \mathscr{H}$ such that $\langle A x, y\rangle_{0}=\left\langle x, A^{*} y\right\rangle_{0}, \forall x, y \in \mathscr{H}$.

This definition is justified by the Riesz representation theorem for real Hilbert space. If $\boldsymbol{A}$ is $\boldsymbol{C}\left(i_{1}\right)$-linear, then $A^{*}$ is just the complex Hilbert space adjoint. If $A$ is linear, the Riesz representation theorem for quaternionic Hilbert space can be used to show that $\langle A x, y\rangle=\left\langle x, A^{*} y\right\rangle, \forall x, y \in \mathscr{H}$. It is easily verified that the mapping $A \mapsto A^{*}$ is a real-linear involution of $\mathscr{L}\left(\mathscr{H}_{0}\right)$ with the property:

$$
(A B)^{*}=B^{*} A^{*}, \quad \forall A, B \in \mathscr{L}\left(\mathscr{H}_{0}\right) .
$$

It should also be noted that, for any quaternion $a$, the adjoint of the multiplication operator $a I$ is $a^{\sigma} I$.

5. Characterisation of real-linear operators. The basic technique is to carry over to operators the decomposition formula given in the following lemma.

LEMmA. Let $\left(i_{1}, i_{2}, i_{3}\right)$ be any right-handed orthonormal triad of vector quaternions. Then the components $q_{0}, q_{1}, q_{2}, q_{3}$ of an arbitrary quaternion $q=\sum_{v=0}^{3} q_{v} i_{v}$ can be found from the matrix expression

$$
\begin{array}{r}
{\left[q_{0}, q_{1}, q_{2}, q_{3}\right]^{T}=Q\left[q,-q i_{1},-q i_{2},-q i_{3}\right]^{T},} \\
\text { where } 4 Q=\left[\begin{array}{cccc}
1 & i_{1} & i_{2} & i_{3} \\
-i_{1} & 1 & i_{3} & -i_{2} \\
-i_{2} & -i_{3} & 1 & i_{1} \\
-i_{3} & i_{2} & -i_{1} & 1
\end{array}\right]
\end{array}
$$

Proof. The $1 \times 4$ quaternion matrix $\left[q-q i_{1}-q i_{2}-q i_{3}\right]^{T}$ can be written in the form

$$
\left[q,-q i_{1},-q i_{2},-q i_{3}\right]^{T}=4 Q\left[q_{0}, q_{1}, q_{2}, q_{3}\right]^{T} .
$$


But it can be shown by direct matrix multiplication that $(4 Q)^{2}=4 I$. Thus

$$
\left[q_{0}, q_{1}, q_{2}, q_{3}\right]^{T}=Q\left[q,-q i_{1},-q i_{2},-q i_{3}\right]^{T}=Q q\left[1,-i_{1},-i_{2},-i_{3}\right]^{T} .
$$

This canonical decomposition formula for quaternions is the inspiration for the main result.

THEOREM 2. Every continuous real-linear operator A mapping a quaternionic Hilbert space $\mathscr{H}$ into a quaternionic Hilbert space $\mathscr{K}$ can be expanded in the form of an arbitrary right-handed orthonormal triad $\left(i_{1}, i_{2}, i_{3}\right)$ of vector quaternions in the form:

$$
A=A_{0}+i_{1} A_{1}+i_{2} A_{2}+i_{3} A_{3},
$$

where $A_{0}, A_{1}, A_{2}, A_{3}$ are continuous linear operators mapping $\mathscr{H}$ into $\mathscr{K}$. Moreover, they are uniquely determined by $A$ and the choice of quaternion basis $\left(i_{1}, i_{2}, i_{3}\right)$.

Proof. The proof is based on a computation which imitates that used to prove the lemma. Let $A: \mathscr{H} \rightarrow \mathscr{K}$ be a continuous real-linear operator and let $\left(i_{1}, i_{2}, i_{3}\right)$ be a right-handed orthonormal triad of vector quaternions. We define the operators $A_{0}, A_{1}, A_{2}, A_{3}$ by the "operator matrix equation"'

$$
\left[A_{0}, A_{1}, A_{2}, A_{3}\right]^{T}=Q A\left[I,-i_{1} I,-i_{2} I,-i_{3} I\right]^{T},
$$

where $I$ denotes the identity operator on $\mathscr{H}$. In other words:

$$
\begin{aligned}
& 4 A_{0}=A-i_{1} A i_{1} I-i_{2} A i_{2} I-i_{3} A i_{3} I, \\
& 4 A_{1}=-i_{1} A-A i_{1} I-i_{3} A i_{2} I+i_{2} A i_{3} I, \\
& 4 A_{2}=-i_{2} A+i_{3} A i_{1} I-A i_{2} I-i_{1} A i_{3} I, \\
& 4 A_{3}=-i_{3} A-i_{2} A i_{1} I+i_{1} A i_{2} I-A i_{3} I .
\end{aligned}
$$

Multiplying (8) on the left by $Q$ gives:

$$
A\left[I,-i_{1} I,-i_{2} I,-i_{3} I\right]^{T}=4 Q\left[A_{0}, A_{1}, A_{2}, A_{3}\right]^{T},
$$

the first row of which is (7). Clearly $A_{0}, A_{1}, A_{2}, A_{3}$ are continuous, and routine computations show that they are linear.

To prove uniqueness, suppose that $A=A_{0}+i_{1} A_{1}+i_{2} A_{2}+i_{3} A_{3}=B_{0}+$ $i_{1} B_{1}+i_{2} B_{2}+i_{3} B_{3}$, where $A_{0}, A_{1}, A_{2}, A_{3}$ are the linear operators defined by (8), and $B_{0}, B_{1}, B_{2}, B_{3}$ are also linear operators. A simple calculation shows that

$$
A\left[I,-i_{1} I,-i_{2} I,-i_{3} I\right]^{T}=4 Q\left[B_{0}, B_{1}, B_{2}, B_{3}\right]^{T}
$$


Combining (9) and (10) and recalling that the quaternion matrix $Q$ is nonsingular, we have

$$
\left[A_{0}, A_{1}, A_{2}, A_{3}\right]^{T}=\left[B_{0}, B_{1}, B_{2}, B_{3}\right]^{T} \text { as required. }
$$

The main result of this theorem can be expressed concisely by $\mathscr{L}\left(\mathscr{H}_{0}, \mathscr{K}_{0}\right)=\left\{\sum_{v=0}^{3} i_{v} A_{v}: A_{v} \in \mathscr{L}(\mathscr{H}, \mathscr{K})\right\}$. The expression $\sum_{v=0}^{3} i_{v} A_{v}$ is called the canonical decomposition of the operator. In the case where $\mathscr{H}=$ $\mathscr{K}$, we see that $\mathscr{L}(\mathscr{H})$ is a real algebra and $\mathscr{L}\left(\mathscr{H}_{0}\right)$ is the quaternion algebra generated by it.

Corollary. $\quad \mathscr{L}\left(\mathscr{H}_{1}\left(i_{1}\right), \mathscr{K}_{1}\left(i_{1}\right)\right)=\left\{A_{0}+i_{1} A_{1}: A_{0}, A_{1} \in \mathscr{L}(\mathscr{H}, \mathscr{K})\right\}$.

Proof. If $A$ is $C\left(i_{1}\right)$-linear, then (8) gives $A_{2}=A_{3}=0$.

Comparison of this result with $(5)$ reveals that the $\omega\left(i_{1}\right)$-linear operators form a proper subset of $\mathscr{L}\left(\mathscr{H}_{1}\left(i_{1}\right), \mathscr{K}_{1}\left(i_{1}\right)\right)$.

We also observe that if $A: \mathscr{H} \rightarrow \mathscr{H}$ is a continuous real-linear operator, then the adjoint $A^{*}$ of $A$ is given by

$$
A^{*}=A_{0}^{*}-i_{1} A_{1}^{*}-i_{2} A_{2}^{*}-i_{3} A_{3}^{*}
$$

Thus $A$ is Hermitian if and only if $A_{0}$ is Hermitian and $A_{1}, A_{2}, A_{3}$ are skewHermitian.

It now remains to investigate the effect on $A_{0}, A_{1}, A_{2}, A_{3}$ of a rotation of the triad $\left(i_{1}, i_{2}, i_{3}\right)$.

THEOREM 3. Let $\left(i_{1}, i_{2}, i_{3}\right)$ and $\left(i_{1}^{\prime}, i_{2}^{\prime}, i_{3}^{\prime}\right)$ be two right-handed orthonormal triads of vector quaternions and let $L=\left[l_{\mu v}\right]$ be the matrix of the threedimensional rotation which transforms the first triad into the second. Let A : $\mathscr{H} \rightarrow \mathscr{K}$ be a continuous real-linear operator and write

$$
A=A_{0}+i_{1} A_{1}+i_{2} A_{2}+i_{3} A_{3}=A_{0}^{\prime}+i_{1}^{\prime} A_{1}^{\prime}+i_{2}^{\prime} A_{2}^{\prime}+i_{3}^{\prime} A_{3}^{\prime} .
$$

Then

$$
\left[A_{0}^{\prime}, A_{1}^{\prime}, A_{2}^{\prime}, A_{3}^{\prime}\right]=\left[A_{0}, A_{1}, A_{2}, A_{3}\right]\left[\begin{array}{c:c}
1 & 0 \\
\hdashline 0 & L
\end{array}\right] .
$$

Proof. By definition of $L$, we have

$$
\left[i_{1}^{\prime} i_{2}^{\prime} i_{3}^{\prime}\right]=\left[\begin{array}{lll}
i_{1} & i_{2} & i_{3}
\end{array}\right] L
$$

Equivalently, since $L$ is a real orthogonal matrix, $\left[i_{1} i_{2} i_{3}\right]=\left[i_{1}^{\prime} i_{2}^{\prime} i_{3}^{\prime}\right] L^{T}$.

$$
A=A_{0}+\sum_{\mu=1}^{3} i_{\mu} A_{\mu}=A_{0}+\sum_{\mu=1}^{3} \sum_{\nu=1}^{3} l_{\mu \nu} i_{\nu}^{\prime} A_{\mu}
$$


But $A=A_{0}^{\prime}+\sum_{v=1}^{3} i_{v}^{\prime} A_{v}^{\prime}$. So, by the uniqueness of the canonical decomposition, $A_{0}^{\prime}=A_{0}$ and $A_{\nu}^{\prime}=\sum_{\mu=1}^{3} l_{\mu \nu} A_{\mu}(\nu=1,2,3)$.

As immediate consequences of this theorem we have

Corollary 1. Let $A=A_{0}+i_{1} A_{1}+i_{2} A_{2}+i_{3} A_{3}=A_{0}^{\prime}+i_{1}^{\prime} A_{1}^{\prime}+i_{2}^{\prime} A_{2}^{\prime}+i_{3}^{\prime} A_{3}^{\prime}$, as in Theorem 3. Then $A_{0}=A_{0}^{\prime}$. Furthermore, if say $i_{1}=i_{1}^{\prime}$, then $A_{1}=A_{1}^{\prime}$.

Corollary 2. Suppose that the operator $A=A_{0}+i_{1} A_{1}+i_{2} A_{2}+i_{3} A_{3}$ is $C(u)$-linear for some unit vector quaternion $u$. Then the operators $A_{1}, A_{2}, A_{3}$ are all real multiples of the same linear operator. Equivalently, $A=A_{0}+u A_{u}$, where $A_{u}$ is a uniquely determined linear operator.

Proof. Choose a right-handed orthonormal triad $\left(i_{1}^{\prime}, i_{2}^{\prime}, i_{3}^{\prime}\right)$ of vector quaternions such that $i_{1}^{\prime}=u$. Then we can write $A=A_{0}+i_{1}^{\prime} A_{u}$. By Corollary $1, A_{u}$ does not depend on the choice of $i_{2}^{\prime}$ and $i_{3}^{\prime}$. Let $L$ be the matrix defined by (11). Then $\left[A_{1}, A_{2}, A_{3}\right]=\left[A_{u}, 0,0\right] L^{T}$, i.e. $A_{1}=l_{11} A_{u}, A_{2}=l_{21} A_{u}, A_{3}=$ $l_{31} A_{u}$.

EXAMPLE. We illustrate our results with a brief discussion of matrix operators. Let $P=\left[p_{j k}\right]$ be any $n \times n$ matrix with quaternion entries and let $P_{L}$ be the operator defined on the $n$-dimensional quaternionic Hilbert space $\mathscr{Q}^{n}$ by multiplying the column vectors of $\mathscr{Q}^{n}$ on the left by $P$-i.e., $y=P_{L} x$ is given by

$$
y_{j}=\sum_{k=1}^{n} p_{j k} x_{k} \quad(j=1, \cdots, n) .
$$

The operator $P_{L}$ is linear if all the elements of $P$ are real, but in general we can only say that $P_{L}$ is a continuous real-linear operator. The canonical decomposition of $P_{L}$ is given by

$$
P_{L}=\left(P_{0}\right)_{L}+i_{1}\left(P_{1}\right)_{L}+i_{2}\left(P_{2}\right)_{L}+i_{3}\left(P_{3}\right)_{L},
$$

where $P_{0}, P_{1}, P_{2}, P_{3}$ are the real matrices obtained by decomposing the entries in $P$. The adjoint of $P_{L}$ is $\left(P^{*}\right)_{L}$, where $P^{*}$ is the transposed conjugate matrix $\left[\left(p_{k j}\right)^{\sigma}\right]$.

It should be mentioned that the matrix $P$ can be made to operate on $\mathscr{Q}^{n}$ in another way-by multiplying the row vectors $x^{T}$ on the right by the transposed matrix $P^{T}$. In other words, we define the operator $P_{R}$ on $\mathscr{Q}^{n}$ by writing $y=P_{R} x$, where $y_{j}=\sum_{k=1}^{n} x_{k} p_{j k}(j=1, \cdots, n)$. The operator $P_{R}$ is linear and is in fact the "orthodox" action on $\mathscr{Q}^{n}$ of a matrix with quaternion entries. That is, up to the present every discussion of matrix operators has used right multiplication by a matrix or, equivalently, has used left multiplication in the context of a right $\mathscr{Q}$-module (see, for example, [2]). Thus the resulting operators have always been linear.

The possibility of constructing a functional calculus for a class of reallinear operators will be discussed in a subsequent paper. 


\section{REFERENCES}

1. J. M. Jauch, Projective representations of the Poincaré group in a quaternionic Hilbert space, Group Theory and its Applications, E. Loebl (editor), Academic Press, New York, 1968, pp. 131-182.

2. O. Teichmüller, Operatoren im Wachsschen Raum, J. Reine Angew. Math. 174 (1935), 73-124.

3. K. Viswanath, Normal operators on quaternionic Hilbert space, Trans. Amer. Math. Soc. 162 (1971), 337-350. MR 44 \#2067.

Department of Mathematics, University of Witwaterstrand, Johannesburg, SOUTH AFrica 